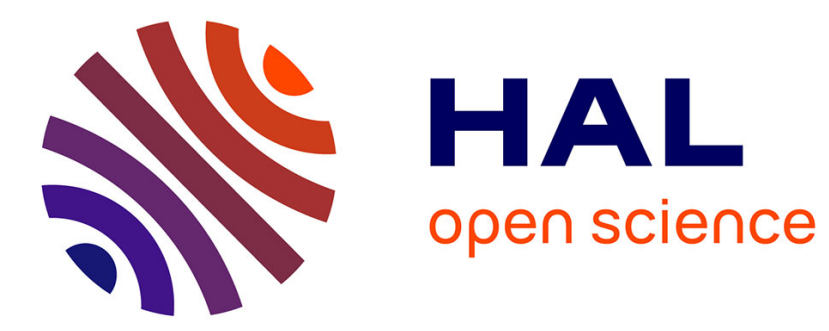

\title{
A real seminorm with square property is submultiplicative
}

\author{
Mohammed El Azhari
}

\section{To cite this version:}

Mohammed El Azhari. A real seminorm with square property is submultiplicative. Indian Journal of Pure and Applied Mathematics, 2012, 43 (4), pp.303-307. 10.1007/s13226-012-0018-z . hal-01303277

\section{HAL Id: hal-01303277 https://hal.science/hal-01303277}

Submitted on 17 Apr 2016

HAL is a multi-disciplinary open access archive for the deposit and dissemination of scientific research documents, whether they are published or not. The documents may come from teaching and research institutions in France or abroad, or from public or private research centers.
L'archive ouverte pluridisciplinaire HAL, est destinée au dépôt et à la diffusion de documents scientifiques de niveau recherche, publiés ou non, émanant des établissements d'enseignement et de recherche français ou étrangers, des laboratoires publics ou privés. 


\title{
A REAL SEMINORM WITH SQUARE PROPERTY IS SUBMULTIPLICATIVE
}

\author{
M.El Azhari
}

Ecole Normale Supérieure, Avenue Oued Akreuch, Takaddoum,

BP 5118, Rabat, Morocco

E-mail: mohammed.elazhari@yahoo.fr

A seminorm with square property on a real associative algebra is submultiplicative.

Key words: real seminorm, seminorm with square property, submultiplicative seminorm.

\section{INTRODUCTION}

It was proved in [3] that every complex seminorm with square property on a commutative algebra is submultiplicative, and it was posed the problem whether this result holds in a noncommutative algebra. This problem was answered in the particular case of Banach algebras [4] and fully resolved in [2], [5] and [7]. The result of [3] holds in the real case. But the results of [2], [4], [5] and [7] don't hold in the real case since we use the Hirschfeld-Zelazko Theorem [6], or its locally bounded version [2], which are not valid in the real case. Using a functional representation theorem [1, Theorem 1], we show that every real seminorm with square property is submultiplicative.

\section{PRELIMINARIES}

Let $A$ be an associative algebra over the field $K=R$ or $C$. A seminorm on $A$ is a function $p: A \rightarrow[0, \infty)$ satisfying $p(a+b) \leq p(a)+p(b)$ for all $a, b$ in $A$ and $p(k a)=|k| p(a)$ for all $a$ in $A$ and $k$ in $K . p$ is a complex seminorm if $K=C$ and $p$ is a real seminorm if $K=R$. $p$ is submultiplicative if $p(a b) \leq p(a) p(b)$ for all $a, b$ in $A$. $p$ satisfies the square property if $p\left(a^{2}\right)=p(a)^{2}$ for all $a$ in $A$. Let $A$ be a real algebra and let a be any element of $A$. The spectrum sp (a) of $a$ in $A$ is defined to be equal to the spectrum of a as an element of the complexification of $A$. If $A$ is unital, then sp $(a)=\left\{s+\right.$ it $\left.\in C,(a-s e)^{2}+t^{2} e \notin A^{-1}\right\}$ for all a in $A$, where $e$ is the unit of $A$ and $A^{-1}$ is the set of all invertible elements in $A$. Let $(A,\|\|$.$) be a real normed$ algebra, the limit $r(a)=\lim \left\|a^{n}\right\|^{1 / n}$ exists for each $a$ in $A$. If $A$ is complete, then $r(a)=\sup \{|v|, v \in \operatorname{sp}(a)\}$ for every $a$ in $A$. 


\section{RESULTS}

Let $(A,\|\|$.$) be a real Banach algebra with unit such that \|a\| \leq m r(a)$ for some positive constant $m$ and all $a$ in $A$. Let $X(A)$ be the set of all nonzero multiplicative linear functionals from $A$ into the noncommutative algebra $H$ of quaternions. For $a$ in $A$ and $x$ in $X(A)$, put $J(a)(x)=x(a)$. For a in $A$, $J(a): X(A) \rightarrow H$ is a map from $X(A)$ into $H . X(A)$ is endowed with the topology generated by $J(a), a \in A$, that is the weakest topology such that all the functions $\mathrm{J}(\mathrm{a}), \mathrm{a} \in \mathrm{A}$, are continuous. By $[1$, Theorem 1$]$, $X(A)$ is a nonempty compact space and the map $J: A \rightarrow C(X(A), H), a \rightarrow J(a)$, is an isomorphism (into), where $C(X(A), H)$ is the real algebra of all continuous functions from $X(A)$ into $H$.

Proposition 3.1. (1) An element $a$ is invertible in $A$ if and only if $J(a)$ is invertible in $C(X(A), H)$, and (2) $\operatorname{sp}(\mathrm{a})=\operatorname{sp}(\mathrm{J}(\mathrm{a}))$ for all $\mathrm{a}$ in $\mathrm{A}$.

Proof. (1) The direct implication is obvious. Conversely, there exists $g$ in $C(X(A), H)$ such that $J(a) g=g J(a)=1$ i.e. $x(a) g(x)=g(x) x(a)=1$ for all $x$ in $X(A)$. Let $T$ be a nonzero irreducible representation of $A$, by the proof of $[1$, Theorem 1$]$ there exists $S: T(A) \rightarrow H$ an isomorphism (into). Since SoT $\in X(A)$ and $0 \neq \operatorname{SoT}(a)=S(T(a))$, it follows that $T(a) \neq 0$. If $a A \neq A$, there exists a maximal right ideal $M$ containing aA. Let $L$ be the canonical representation of $A$ on $A / M$ which is nonzero and irreducible, also $L(a)=0$ since $a A$ is included in $M$, contradiction. Then $a A=A$ and by the same $A a=A$. There exist $b, c$ in $A$ such that $a b=c a=e(e$ is the unit of $A)$. We have $c=c(a b)=(c a) b=b$, so $a$ is invertible in $A$.

(2) $\mathrm{s}+$ it $\in \mathrm{sp}(\mathrm{a})$ iff $(\mathrm{a}-\mathrm{se})^{2}+\mathrm{t}^{2} \mathrm{e} \notin \mathrm{A}^{-1}$

$$
\begin{aligned}
& \text { iff } J\left((a-s e)^{2}+t^{2} e\right) \notin C(X(A), H)^{-1} \text { by }(1) \\
& \text { iff }(J(a)-s J(e))^{2}+t^{2} J(e) \notin C(X(A), H)^{-1} \\
& \text { iff } s+\text { it } \in s p(J(a)) .
\end{aligned}
$$

Theorem 3.2. Let $A$ be a real associative algebra. Then every seminorm with square property on $A$ is submultiplicative.

Proof. If $A$ is commutative, see [3, Theorem 1]. If $A$ is noncommutative, let $p$ be a seminorm with square property on $A$. By [5] or [7], there exists $m>0$ such that $p(a b) \leq m p(a) p(b)$ for all $a, b$ in $A$. $\operatorname{Ker}(p)$ is a two sided ideal in $A$, the norm $|$.$| on the quotient algebra A / \operatorname{Ker}(p)$, defined by $|a+\operatorname{Ker}(p)|=p(a)$ is a norm with square property on $A / \operatorname{Ker}(p)$. Define $\|a+\operatorname{Ker}(p)\|=m|a+\operatorname{Ker}(p)|$ for all $a$ in $A$. Let $a, b$ in $A,\|a b+\operatorname{Ker}(p)\|=m|a b+\operatorname{Ker}(p)| \leq m^{2}|a+\operatorname{Ker}(p)||b+\operatorname{Ker}(p)|=\|a+\operatorname{Ker}(p)\|\|b+\operatorname{Ker}(p)\|$. $(A / \operatorname{Ker}(p),\|\|$.$) is a real normed algebra. Let a$ in $A,\left\|a^{2}+\operatorname{Ker}(p)\right\|=m\left|a^{2}+\operatorname{Ker}(p)\right|=m|a+\operatorname{Ker}(p)|^{2}=$ $m^{-1}(m|a+\operatorname{Ker}(p)|)^{2}=m^{-1}\|a+\operatorname{Ker}(p)\|^{2}$. The completion $B$ of $(A / \operatorname{Ker}(p),\|\|$.$) satisfies also the$ property $\left\|b^{2}\right\|=m^{-1}\|b\|^{2}$ for all $b$ in $B$, and consequently $\left\|b^{2^{n}}\right\|^{2^{-n}}=m^{2^{-n}-1}\|b\|$ for all $b$ in $B$ and $n$ in $N^{*}$, then $r(b)=m^{-1}\|b\|$ i.e. $\|b\|=m r(b)$. We consider two cases.

$B$ is unital: $B y[1$, Theorem 1$], X(B)$ is a nonempty compact space and the map $\mathrm{J}: B \rightarrow C(X(B), H)$ is an isomorphism ( into). $C(X(B), H)$ is a real Banach algebra with unit under the supnorm $\|.\|_{s}$. By 
Proposition 3.1, $r(b)=r(J(b))$ for all $b$ in $B$. Let $b$ in $B,\|b\|=m r(b)=m r(J(b))=m\|J(b)\|_{s}$ since the supnorm satisfies the square property. Then $\|J(b)\|_{s}=m^{-1}\|b\|=|b|$ for all $b$ in $A / \operatorname{Ker}(p)$, so $|$.$| is$ submultiplicative on $A / \operatorname{Ker}(p)$ i.e. $p$ is submultiplicative.

$B$ is not unital: Let $B_{1}$ be the algebra obtained from $B$ by adjoining the unit. By the same proof of [6, Lemma 2], there exists a norm $\mathrm{N}$ on $\mathrm{B}_{1}$ such that

(i) $\quad\left(B_{1}, N\right)$ is a real Banach algebra with unit

(ii) $\quad \mathrm{N}(\mathrm{b}) \leq \mathrm{m}^{3} \mathrm{r}_{\mathrm{B}_{1}}$ (b) for all $\mathrm{b}$ in $\mathrm{B}_{1}$

(iii) $\quad \mathrm{N}$ and $\|$.$\| are equivalent on \mathrm{B}$.

By $[1$, Theorem 1$], X\left(B_{1}\right)$ is a nonempty compact space and the map J: $B_{1} \rightarrow C\left(X\left(B_{1}\right), H\right)$ is an isomorphism ( into). Let $b$ in $B,\|b\|=m r_{B}(b)=m r_{B_{1}}(b)$ by (iii)

$=\mathrm{m} \mathrm{r}(\mathrm{J}(\mathrm{b}))$ by Proposition 3.1

$=\mathrm{m}\|\mathrm{J}(\mathrm{b})\|_{\mathrm{s}}$ by the square property of the supnorm.

Then $\|J(b)\|_{s}=m^{-1}\|b\|=|b|$ for all $b$ in $A / \operatorname{Ker}(p)$, so $|$.$| is submultiplicative on A / \operatorname{Ker}(p)$ i.e. $p$ is submultiplicative.

\section{REFERENCES}

1. M.Abel and K.Jarosz, Noncommutative uniform algebras, Studia Math., 162 (2004), 213-218.

2. J.Arhippainen, On locally pseudoconvex square algebras, Publicacions Matemàtiques, 39 (1995), 89-93.

3. S.J.Bhatt and D.J.Karia, Uniqueness of the uniform norm with an application to topological algebras, Proc. Amer. Math. Soc., 116 (1992), 499-504.

4. S.J.Bhatt, A seminorm with square property on a Banach algebra is submultiplicative, Proc. Amer. Math. Soc., 117 (1993), 435-438.

5. H.V.Dedania, A seminorm with square property is automatically submultiplicative, Proc. Indian. Acad. Sci ( Math. Sci.), 108 (1998), 51-53.

6. R.A.Hirschfeld and W.Zelazko, On spectral norm Banach algebras, Bull. Acad. Polon. Sci. Sér. Sci. Math. Astronom. Phys., 16 (1968), 195-199.

7. Z.Sebestyén, A seminorm with square property on a complex associative algebra is submultiplicative, Proc. Amer. Math. Soc., 130 (2001), 1993-1996. 
